OPEN

SUBJECT AREAS:

SOLAR CELLS

POLYMERS

CHEMICAL PHYSICS

SPECTROSCOPY

Received

17 April 2013

Accepted

7 June 2013

Published

25 June 2013

Correspondence and requests for materials should be addressed to

G.L. (guglielmo.

lanzani@iit.it)

\section{Ultrafast Energy Transfer in Ultrathin Organic Donor/Acceptor Blend}

\author{
Ajay Ram Srimath Kandada ${ }^{1,2}$, Giulia Grancini ${ }^{2}$, Annamaria Petrozza², Stefano Perissinotto², \\ Daniele Fazzi ${ }^{2}$, Sai Santosh Kumar Raavi ${ }^{2} \&$ Guglielmo Lanzani ${ }^{1,2}$
} 'Dipartimento di Fisica, Piazza Leonardo da Vinci 32, 20133 Milano, Italy, ${ }^{2}$ Center for Nano Science and Technology @ PoliMi,
Istituto Italiano di Tecnologia, via Pascoli 70/3 20133 Milano, Italy.

It is common knowledge that poly(3-hexylthiophene) $(\mathrm{P} 3 \mathrm{HT}) /[6,6]$-phenyl-C61-butyric acid methyl ester (PCBM) blend, a prototype system for bulk heterojunction (BHJ) solar cells, consists of a network of tens of nanometers-large donor-rich and acceptor-rich phases separated by extended finely intermixed border regions where PCBM diffuse into P3HT. Here we specifically address the photo-induced dynamics in a $10 \mathrm{~nm}$ thin P3HT/PCBM blend that consists of the intermixed region only. Using the multi-pass transient absorption technique (TrAMP) that enables us to perform ultra high sensitive measurements, we find that the primary process upon photoexcitation is ultrafast energy transfer from P3HT to PCBM. The expected charge separation due to hole transfer from PCBM to P3HT occurs in the $100 \mathrm{ps}$ timescale. The derived picture is much different from the accepted view of ultra-fast electron transfer at the polymer/PCBM interface and provides new directions for the development of efficient devices.

$\mathrm{D}$ ue to the tremendous surge of interest in organic photovoltaics, the photophysics at the electron donor/ acceptor (D/A) organic interface is a highly debated topic in material science. One of the most extensively investigated D/A blend is made of poly(3-hexylthiophene) ( $\mathrm{P} 3 \mathrm{HT}$ ), as the donor component, mixed with phenyl-C61-butyric acid methyl ester (PCBM), as the acceptor. This blend provides solar cells with $85 \%$ Internal Quantum Efficiency ${ }^{1}$ and power conversion efficiency of around $5 \%^{2}$. Such high quantum yield of charge photogeneration with respect to pure organic semiconductors results from blending the two components into a BHJ. The D/A components are deposited from common solution and tend to segregate over tens of nanometers forming PCBM-rich clusters and crystalline domains of $\mathrm{P} 3 \mathrm{HT}^{1-6}$. The two phases however are not separated by sharp interfaces, because substantial intermixing takes place in the border region defined by the extent of diffusion of PCBM molecules into $\mathrm{P}^{3} \mathrm{HT}^{7}$. In this intermixed region, the two components are finely dispersed at the molecular level and have been clearly observed in bi-layer structures ${ }^{7}$. Here we address the photophysical processes happening only at the intermixed border region by probing an ultrathin film with thickness much below the PCBM diffusion length.

By and large, charge transfer is regarded as the fundamental event occurring upon donor photo-excitation in the $\mathrm{BHJ}^{8,9}$ while energy transfer is generally ignored, at least at early times. However the proximity of D and A molecules in the blend may in principle also lead to electronic excitation energy transfer. Indeed this has been reported for a few systems prepared ad hoc, such as D/A bilayers separated by an interlayer that hampers charge separation ${ }^{10-12}$ or in chemically bound dyads, wherein the $\mathrm{D}$ and $\mathrm{A}$ are in a fixed positions with respect to each other $^{13}$. Both these cases are thus modifications of the standard BHJ, where essentially the wavefunction overlap is largely reduced. On the contrary, there are no experimental evidences of electronic excitation energy transfer as primary event in standard $\mathrm{BHJ}$ systems. Based on photoluminescence (PL) quenching experiments in D/A blends, it was proposed that energy transfer dominates charge transfer only after 5 ps from the excitation ${ }^{14}$. The suggested picture is that close pairs undergo charge transfer, while distant pairs may first interact by energy transfer. Förster radius, the measure of the electronic coupling strength between $\mathrm{D}$ and $\mathrm{A}$, has been evaluated for the prototypical systems such as $\mathrm{P} 3 \mathrm{HT} / \mathrm{PCBM}$ in the range between 1 and $5 \mathrm{~nm}^{11,13,15}$. Why electronic excitation energy transfer appears only in special situations and why charge transfer overwhelms it in $\mathrm{BHJ}$, remains an open question.

In this work we study the photoexcitation dynamics of the intermixed P3HT/PCBM region obtained from a nominal $5 / 5 \mathrm{~nm}$ diffuse bi-layer. Contrary to the expectations, we demonstrate that ultrafast electronic energy transfer between donor and acceptor is the primary process occurring upon photoexcitation. Subsequent hole transfer from PCBM to the polymer takes place on the 100 ps time scale, resulting in the expected charge separation. 
A thin P3HT layer of about $5 \mathrm{~nm}$ has an optical density of about $2 \times 10^{-2}$ (see Supplementary Information (S.2) for thin film preparation and characterization). This value is far too low to have detectable signal above the shot noise limit in a standard pumpprobe system. To overcome such a limitation, we propose a novel Transient Absorption by Multiple Pass (TrAMP) technique, that enables us to perform measurements on samples with optical densities as low as $10^{-2}$. This technique draws its inspiration from absorption spectroscopy of gases where high sensitivities are achieved by increasing the optical path length by multiple reflections of the beam in the gas chamber ${ }^{16}$. The same principle is extended to transient absorption spectroscopy by passing the pump and probe pulses multiple times, keeping the time delay between them constant. In this way, the pump-probe signals can be amplified by a factor proportional to the number of passes through the sample (see Supplementary Information (S.1) for more details).

Figure 1a shows the schematic lay-out of the TrAMP setup while Figure $1 b$ demonstrates the concept and validity of our approach. It shows the TrAMP kinetics collected at various numbers of passes in a $15 \mathrm{~nm}$ thin film of $\mathrm{P} 3 \mathrm{HT}$ on a $250 \mu \mathrm{m}$ fused silica substrate. The pump is at $400 \mathrm{~nm}$ and the probe wavelength tuned at $680 \mathrm{~nm}$.
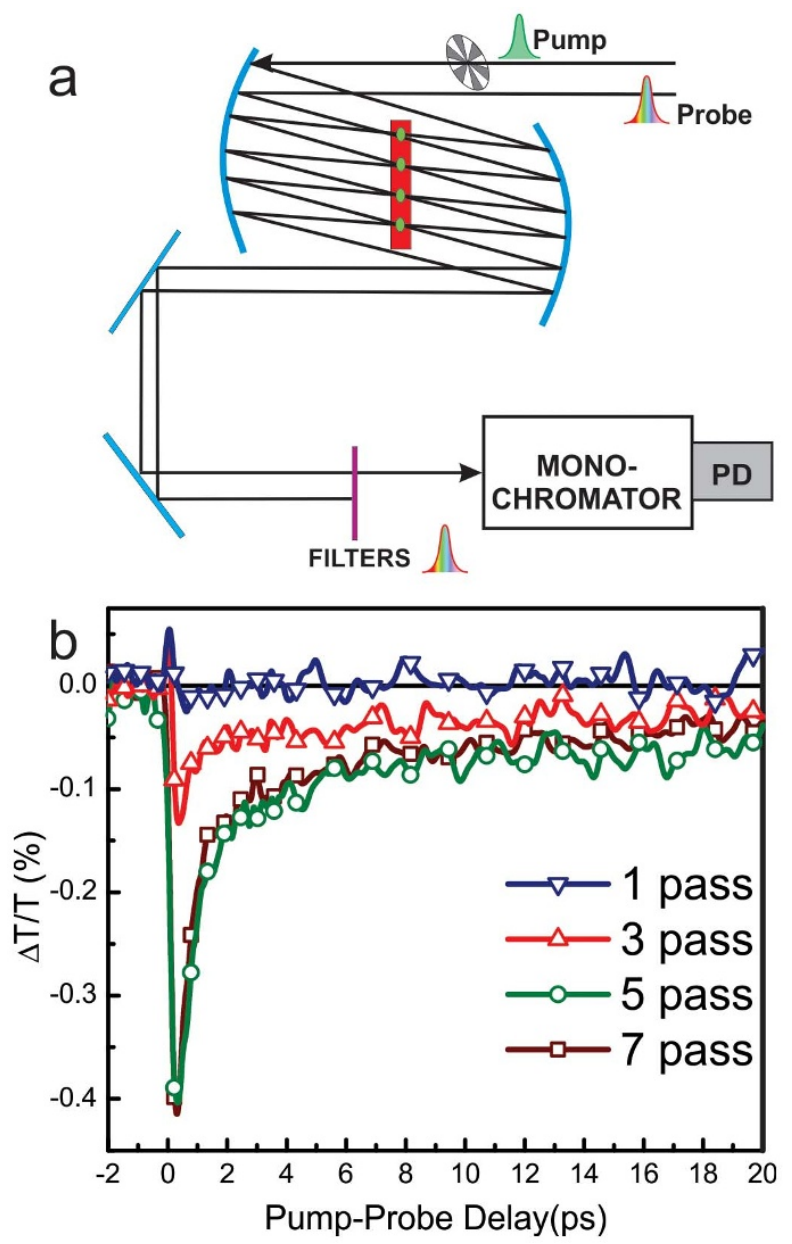

Figure 1 | Transient Absorption by Multiple Pass(TrAMP).

(a) Experimental scheme of TrAMP system. The Modulated pump beam and the probe beam are made to pass through the sample several times within the misaligned cavity made of the two curved mirrors. The sample is placed at the focus of the cavity. A set of filters and a monochromator are used to remove the pump before sending the probe beam to the detection system. (b) TrAMP kinetics collected by increasing the number of passes. The sample is $15 \mathrm{~nm}$ film of P3HT $(\mathrm{OD}=0.1)$, the pump is at $400 \mathrm{~nm}$ and the probe at $680 \mathrm{~nm}$.
Standard pump probe (single pass) cannot detect any differential transmission changes in such a thin film. However, by the multiple pass scheme, the TrAMP technique provides an efficient amplification of the pump-probe signal, that is shown growing at each pass, while keeping the kinetic itself intact. This also demonstrates that possible effects on the time resolution, such as group velocity delay between pump and probe and pulse broadening, are negligible in our experiment, even in the worst condition of large spectral separation between the pump and the probe wavelength. By appropriate group velocity mismatch calculations (see Supplementary Information for details), it can be shown that the expected time resolution of the experiment is within 200 to $400 \mathrm{fs}$ after 15 passes, depending on the pump-probe wavelengths. After certain number of passes, signal amplification reaches a saturation level that can be attributed to the almost complete absorption of the pump. The threshold number of passes depends on the optical density of the sample and for our bilayers this number is between 10 and 15 and all the experiments were conducted within this range. The pump wavelength for all the experiments reported here was tuned to $520 \mathrm{~nm}$. An input energy of $10 \mathrm{~nJ}$ per pulse was employed. The spot-size varies for each pass in TrAMP between $100 \mu \mathrm{m}$ and $200 \mu \mathrm{m}$. This will correspond to a maximum fluence of about $30 \mu \mathrm{J} / \mathrm{cm}^{2}$.

In order to investigate the intermixed D/A system avoiding large phase segregation, we first deposit a $5 \mathrm{~nm}$ P3HT layer on fused-silica substrate and then we spin coat the PCBM on top from an orthogonal solvent ${ }^{7}$ with a nominal thickness of $5 \mathrm{~nm}$. The absorption spectrum of the sample is a linear combination of the absorption spectrum of the two components with an OD of 0.02 at the absorption peak (Figure 2a). Because the P3HT film thickness is smaller than the PCBM molecule diffusion length, PCBM almost fully penetrate the polymer and we obtain a layer of P3HT doped with $\mathrm{PCBM}^{17}$ (see cartoon in Figure 2b). This is also suggested by simple AFM analysis performed on the bilayer (see AFM characterization in figure S3 of the Supplementary Information).

\section{Results}

Figure $3 a$ shows the transient absorption $(\Delta \mathrm{T} / \mathrm{T})$ spectra of $5 \mathrm{~nm}$ thin P3HT film as a control experiment. The TrAMP spectrum and its temporal evolution are consistent with the one reported in literat$u_{r e}{ }^{18}$. At wavelengths shorter than $620 \mathrm{~nm}$ it exhibits a positive signal due to ground state bleaching (GSB). At probe wavelengths greater than $620 \mathrm{~nm}$ it shows a negative photo-induced absorption (PA) signal. A comparison with dynamics, as reported in Figure $3 c$, and a rather extended set of experimental and computational data reported in literature helps in disentangling the contributions beyond spectral overlap ${ }^{19,20}$. Two species can be identified at early time: singlet excitons and polaron pairs (PP) as assigned by Korovyanko et $\mathrm{al}^{18}$. The fast decaying PA band appearing at $650 \mathrm{~nm}$ is assigned to geminate recombination of PP. The longer lived signal is due to singlet states, with some contribution from polarons escaping the geminate recombination as reported in the earlier TA studies on $\mathrm{P} 3 \mathrm{HT}^{8,9,18}$.

The $\Delta \mathrm{T} / \mathrm{T}$ spectrum of the $\mathrm{P} 3 \mathrm{HT} / \mathrm{PCBM}$ intermixed layer is shown in Figure $3 b$, and selected time traces are reported in Figure $3 d$. At a first glance, the early spectrum resembles the pure P3HT spectrum, though with a remarkable reduction in the amplitude of GSB with respect to the PA. Within 2 ps, both the P3HT GSB and the PA band centered at $650 \mathrm{~nm}$ decay to zero with similar kinetics, giving rise to a negative signal extending on the whole experimental range. Notably the $580-620 \mathrm{~nm}$ spectral region that corresponds to $\mathrm{P} 3 \mathrm{HT}$ ground state absorption shows null or negative signal. This indicates that P3HT is weakly or not excited anymore. The broad PA should correspond to an excited state population that is not perceived by the ground state of the polymer. The charge transfer process cannot explain such an observation: upon charge transfer (CT) and hole-polaron formation, the polymer GSB 

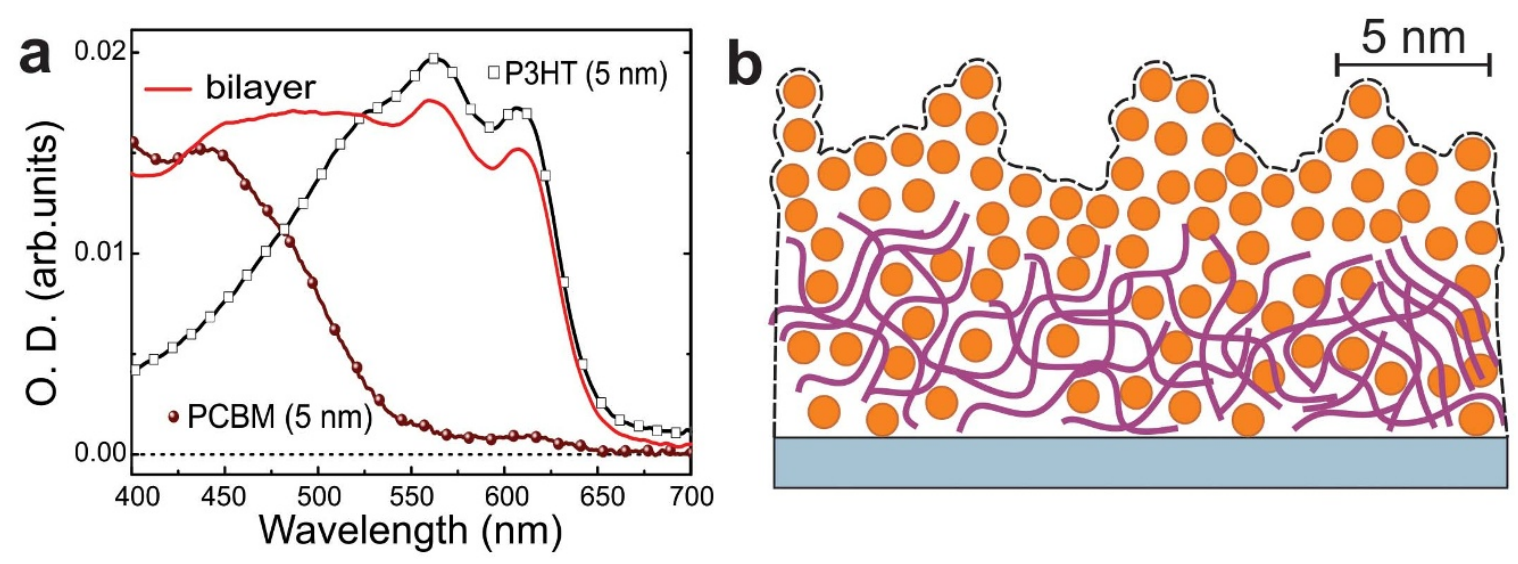

Figure $2 \mid$ Bilayer characterization. (a) Illustration of the morphology of the $5 \mathrm{~nm}$ bilayer of P3HT/PCBM used in present experiment. (b) Absorption spectrum of $5 \mathrm{~nm}$ films of P3HT and PCBM and the bilayer.

stays constant due to the persistent depletion of the ground state population.

Our observation can however be explained by electronic energy transfer from P3HT to PCBM. Accordingly, P3HT GSB would decay and a new PA due to $\left(\mathrm{S}_{\mathrm{n}} \leftarrow \mathrm{S}_{1}\right)$ in PCBM would appear (see Supplementary Information (S.4) for quantum chemically computed $\mathrm{S}_{\mathrm{n}} \leftarrow \mathrm{S}_{1}$ transitions in PCBM). As a reference, $\Delta \mathrm{T} / \mathrm{T}$ spectrum of pristine PCBM, measured upon resonant excitation $(400 \mathrm{~nm})$, is
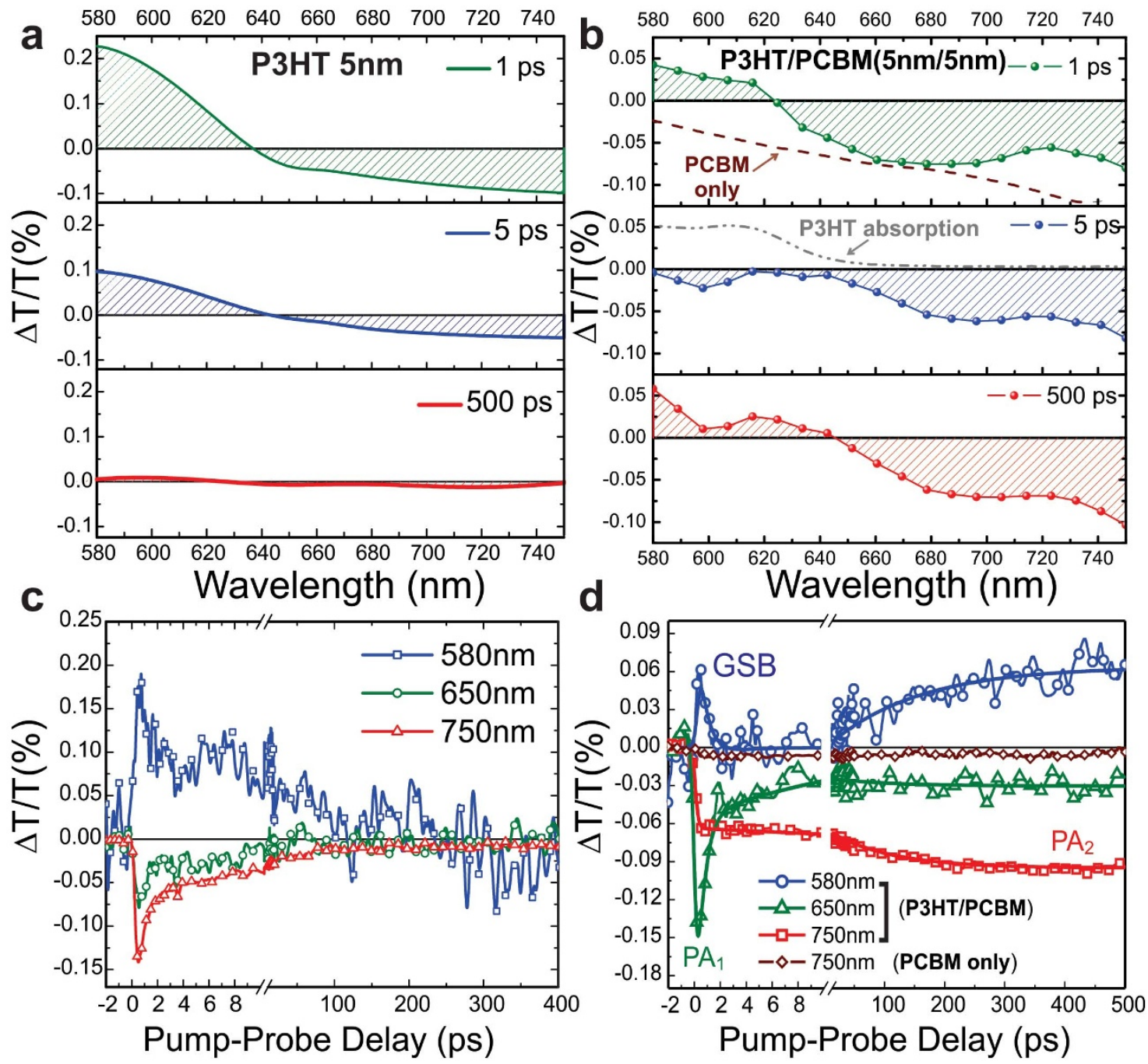

Figure 3 Ultrafast dynamics in the ultrathin P3HT/PCBM bilayers. (a) TrAMP spectra and (c) the associated kinetics of a control film of P3HT with a thickness of $5 \mathrm{~nm}$. (b) TrAMP spectra of P3HT/PCBM $(5 \mathrm{~nm} / 5 \mathrm{~nm})$ bilayer at pump-probe delays of $1 \mathrm{ps,} 5$ ps and 500 ps. The TA spectrum of PCBM pumped at $400 \mathrm{~nm}$ is shown in the $1 \mathrm{ps}$ window. The absorption of $\mathrm{P} 3 \mathrm{HT}$ is also shown. (d) The associated TrAMP kinetics of the bilayer taken at $\lambda_{\text {probe }}=$ $580 \mathrm{~nm}(\mathrm{GSB})$ and at $650 \mathrm{~nm}$ and $750 \mathrm{~nm}$ (PA). Also plotted is the kinetic at $\lambda_{\text {probe }}=750 \mathrm{~nm}$ of a $5 \mathrm{~nm}$ PCBM film. The Pump pulse for all the measurements is tuned to $520 \mathrm{~nm}$. 
reported in Figure 3b. It is a broad photoinduced absorption band, extending over the whole experimental range. This fits reasonably well with our observation, supporting our explanation. It is fair to note a discrepancy between the lineshape of the pure PCBM PA band and our data, in the $580-620 \mathrm{~nm}$ spectral region. This can be ascribed to a residual GSB of the polymer, indicating that following polymer photoexcitation there is a branching between electronic energy transfer and charge transfer towards PCBM, here in favor of the former.

Concominant excitation of PCBM, when we excite the P3HT, should also be evaluated as a possible explanation for the reported experimental results. At this purpose we studied a $5 \mathrm{~nm}$ thick PCBM film, with a similar optical density of the intermixed sample, pumping at $520 \mathrm{~nm}$. The plot in Figure $3 d$ contains the time trace at $750 \mathrm{~nm}$, assigned to $P C B M S_{n} \leftarrow S_{1}$ PA peak (see Supplementary Information Figure S4 and S5). The signal amplitude, without any normalization, clearly shows that the role of direct PCBM excitation is negligible. We infer that direct photo-excitation of PCBM contributes less than $10 \%$ of the signal measured in the P3HT/PCBM sample.

The electronic energy transfer occurs on a time scale faster than our resolution ( $\approx 200 \mathrm{fs}$ ). The broad PA band assigned to PCBM is formed during pump excitation and it is present from the very beginning in our transient spectra. This explains the different ratio between GSB and PA in the P3HT/PCBM sample with respect to the one in pristine $\mathrm{P} 3 \mathrm{HT}$.

On the longer timescale, we observe a build-up of the GSB with a time constant of 130 ps along with a correlated increase in the PA band centered at $750 \mathrm{~nm}$. The return of GSB in transient spectra is a rather unusual phenomenon that is associated to back charge transfer following the initial energy transfer in D-A dyads of different type $^{13,21,22}$. Here it can be understood as the generation of polaron states in P3HT via hole-transfer from PCBM. Though the absorption bands of $\mathrm{P}_{3} \mathrm{HT}^{+}$and PCBM singlet overlap, due to higher oscillator strength of the polaron (see Supplementary Information S.4), its contribution dominates the dynamics. The overlap of the bands can also explain the apparent slower growth rate in the polaron PA with respect to the GSB. This is also confirmed by the slow PA growth at $900 \mathrm{~nm}$ (see Figure 4a) after the initial PP recombination, and this will be discussed in detail later. In neat P3HT the dominant contribution at $900 \mathrm{~nm}$ is the $\left(\mathrm{S}_{\mathrm{n}} \leftarrow \mathrm{S}_{1}\right)$ transition (Figure $4 b$ ), but in the ultrathin diffuse layer such a contribution is absent, consistent with the conjectured electronic energy transfer.

Hence, in the considered molecularly-mixed morphology, the photo-physical picture for charge generation can be interpreted as a two-step process. First, electronic energy transfer happens, as the dominant process, from $\mathrm{P} 3 \mathrm{HT}$ to $\mathrm{PCBM}\left(\mathrm{D}^{*}+\mathrm{A} \rightarrow \mathrm{D}+\mathrm{A}^{*}\right)$; then it is followed by a hole transfer from the excited PCBM back to P3HT, $\left(A^{*}+D \rightarrow D^{+}+A^{-}\right)$.

\section{Discussion}

The phenomenon of ultrafast energy transfer before charge transfer in systems commonly utilized in organic $\mathrm{BHJ}$ solar cells is puzzling and contradicting common beliefs. In order to further test our interpretation we performed experiments on tri-layered samples where charge transfer can be effectively blocked by inserting a large bandgap thick interlayer of Poly-Vinyl Acetate (PVA) between P3HT and PCBM. Measurements on the tri-layer films are reported with thin $(<5 \mathrm{~nm})$ and thick $(\gg 10 \mathrm{~nm})$ PVA layers (Figures $5 b$ and $5 d$ respectively). When the PVA layer is thin the GSB of P3HT fully decays to zero in 2 ps, again leaving a PA without GSB for longer time delays. The lack of GSB is consistent with ultrafast electronic energy transfer even in presence of the thin PVA interlayer. We can set an upper limit for the time scale of the process that is again within the excitation process, about $200-300$ fs. The steady PA is assigned to $S_{n} \leftarrow S_{1}$ PCBM electronic transition. In presence of the interlayer, however, there is neither delayed build up of GSB nor PA at longer time. We suppose that because of the very large surface roughness, there is no substantial reduction in P3HT-PCBM average distance when a thin interlayer is deposited, preserving the dipole-dipole electronic energy transfer, while the charge transfer is suppressed by the lack of wavefunction overlap. When the interlayer is thick (Figure $5 d$ ), pure P3HT dynamics, similar to Figure $3 c$ can be observed. In particular there is a longer lived GSB due to the singlet
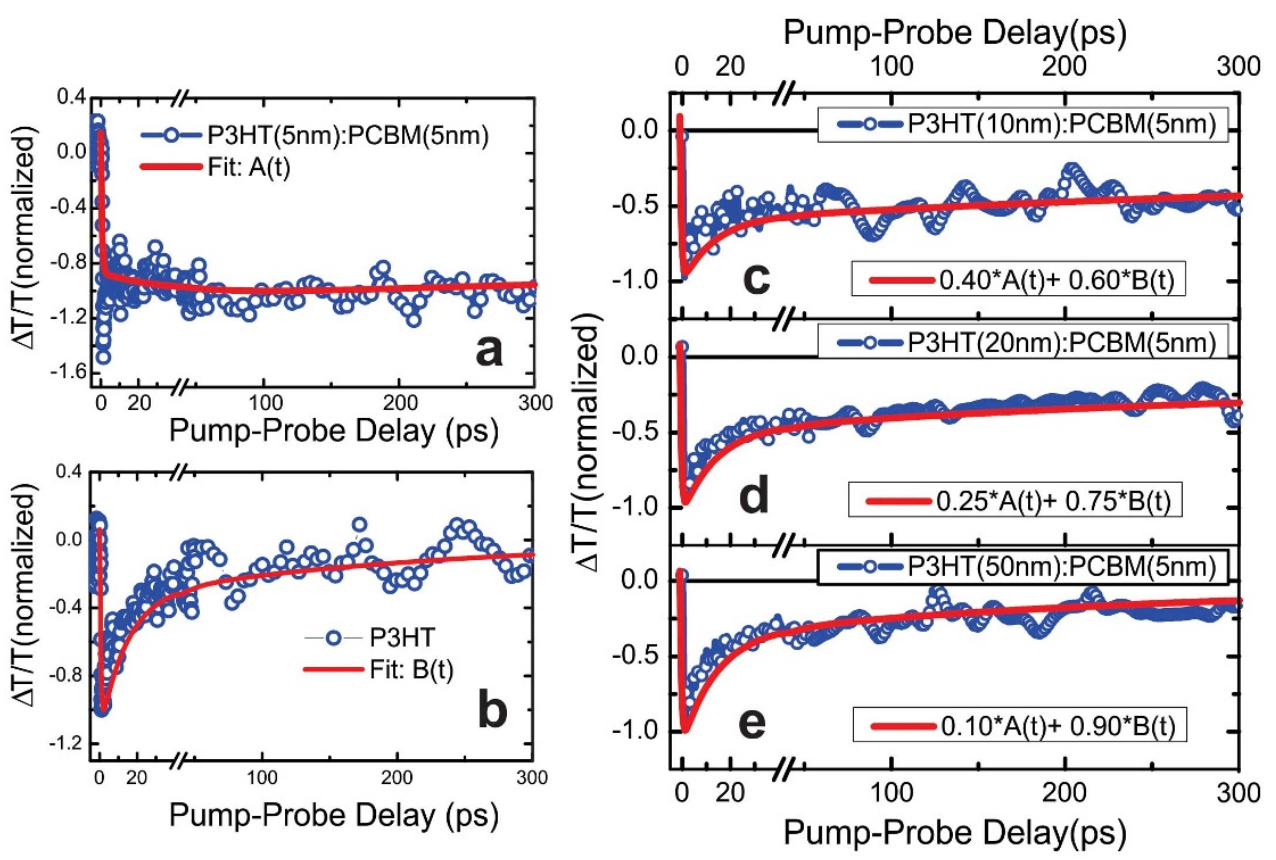

Figure $4 \mid$ Thickness dependent TrAMP dynamics. (a) TrAMP kinetic at $\lambda_{\text {probe }}=900 \mathrm{~nm}$ for $5 \mathrm{~nm} / 5 \mathrm{~nm}$ bilayer of P3HT/PCBM. A multiexponential fit $(\mathrm{A}(\mathrm{t}))$ is also shown. (b) Similar kinetic and the fit $(\mathrm{B}(\mathrm{t}))$ for a $5 \mathrm{~nm}$ film of P3HT. TrAMP kinetics for the bilayers with $10 \mathrm{~nm}(\mathrm{c}), 20 \mathrm{~nm}(\mathrm{~d})$ and $50 \mathrm{~nm}$ (e) thicknesses of P3HT with a constant $5 \mathrm{~nm}$ layer of PCBM on the top. Also shown are the fits obtained by a linear superposition of A(t) and B(t) in each of the three cases. 
Pump-Probe Delay(ps)
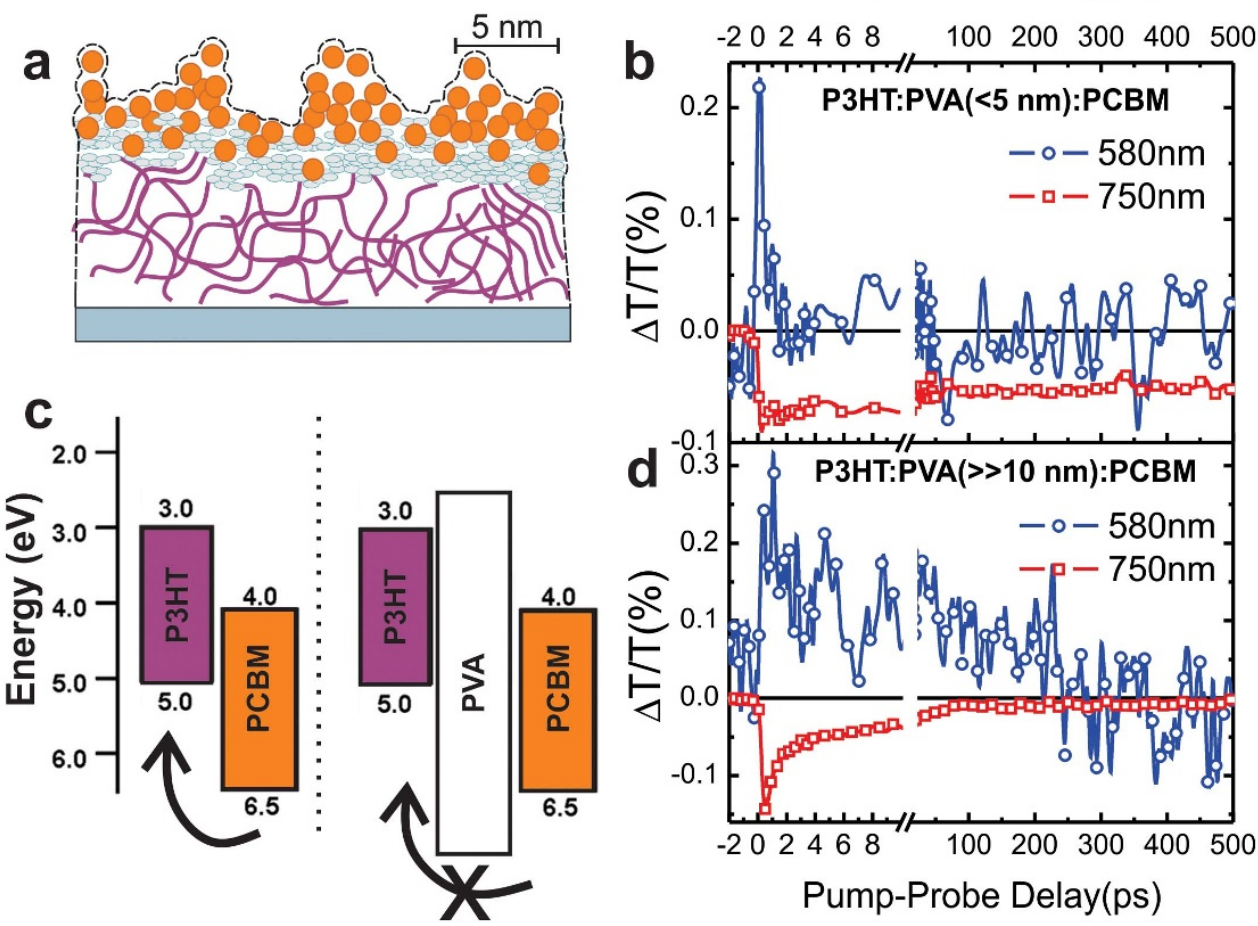

Figure 5 | Ultrafast dynamics in the trilayers of P3HT, PVA and PCBM. (a) Illustration of the possible morphology of the bilayer with a 5 nm interlayer of PVA. (b) TrAMP kinetics at $580 \mathrm{~nm}(\mathrm{GSB})$ and $750 \mathrm{~nm}$ (PA) in the bilayer with a thin $(<5 \mathrm{~nm})$ interlayer of PVA and d) with a thick $\left(\gg 10 \mathrm{~nm}\right.$ ) layer. (c) The energy level scheme ${ }^{24}$ of P3HT/PCBM with the hole transfer pathway from PCBM to P3HT, which is blocked with the introduction of PVA.

states. This confirms that both electronic energy transfer and charge transfer can be suppressed by a thick interlayer.

In the standard $\mathrm{BHJ}$ the intermixed doped region is in between the donor and acceptor clusters ${ }^{23}$. In order to include exciton diffusion into our picture, we perform TrAMP measurements on $\mathrm{P} 3 \mathrm{HT} /$ PCBM bilayers by increasing the thickness of the P3HT layer. Four samples are considered where-in $5 \mathrm{~nm}, 10 \mathrm{~nm}, 20 \mathrm{~nm}$ and $50 \mathrm{~nm}$ thick P3HT films are covered with $5 \mathrm{~nm}$ of PCBM. Each of these samples simulates the spatial extent for exciton diffusion in $\mathrm{P} 3 \mathrm{HT}$ rich domains in a $\mathrm{BHJ}$. The probe wavelength in these experiments is tuned to $900 \mathrm{~nm}$, to have a signature of singlet exciton absorption in P3HT. As discussed earlier however, other species may also contribute at this wavelength.

The $5 / 5 \mathrm{~nm}$ bilayer shows an instantaneous PA that reduces by only a few percent within 2 ps, it stays flat and then it increases in 100 ps time scale (see Figure 4a). According to our model this signal contains contributions from PP recombination at initial time, and from P3HT polarons at longer delays. In Figure $4 b$ we show the signal obtained from a pure $5 \mathrm{~nm}$ P3HT film. The samples in Figures $4 a, b$ may represent the two distinct morphologies present in a $\mathrm{BHJ}$ : the well intermixed $\mathrm{P} 3 \mathrm{HT} / \mathrm{PCBM}$ interface and the pristine $\mathrm{P} 3 \mathrm{HT}$ domain. The other three samples $(10 / 5,20 / 5$ and 50/5 nm) show intermediate behaviors, that can be simulated as a linear combination of the traces in $4 a$ and $4 b$ plots. This is shown by first fitting multi-exponential functions, $\mathrm{A}(\mathrm{t})$ and $\mathrm{B}(\mathrm{t})$, to the time traces in Figures $4 a, b$ respectively. Linear combination of $\mathrm{A}(\mathrm{t})$ and $\mathrm{B}(\mathrm{t})$ reproduces quite well all the other data, enabling us to infer a relative weight of the two components in each of the samples. The sample with $10 \mathrm{~nm}$ P3HT (Figure 4c) shows almost equal contribution from the pristine phase and the intermixed region. The sample with $20 \mathrm{~nm}$ thick P3HT (Figure 4d) shows 25\% contribution from the intermixed region and the as above $50 \mathrm{~nm}$ sample (Figure 4e) shows less than $10 \%$ contribution. The interface specific dynamics are perceivable only when the thickness of the P3HT layer is less than $10 \mathrm{~nm}$. This length scale stands for the balance between the electronic energy transfer and the exciton diffusion limited processes ${ }^{25}$. In a standard $\mathrm{BHJ}$ blend such dynamics are averaged out in the pump probe measurements and due to the spectral overlap and the consequent congestion of signals, charge transfer seems the dominant event.

To further support the proposed electronic energy transfer (EET) mechanism occurring at the P3HT/PCBM molecular interface on an ultrafast time scale we consider, in their simplest forms, the transfer rate equations ruling EET and CT processes ${ }^{26,27}$, reported in the Equations 1 and 2 respectively:

$$
k_{E E T}=k_{\text {Förster }}+k_{\text {Dexter }}=\frac{2 \pi}{\hbar}\left(V_{C}^{2}+V_{D}^{2}\right) J
$$

and

$$
k_{c t}=\frac{2 \pi}{\hbar} V_{C T}^{2}\left\{\left(\frac{1}{4 \pi \lambda k_{B} T}\right)^{\frac{1}{2}} \exp \left(-\frac{(\Delta G+\lambda)^{2}}{4 \lambda k_{B} T}\right)\right\}
$$

In Equation $1, V_{C}$ and $V_{D}$ are the electronic coupling matrix elements for Förster and Dexter mechanism ${ }^{28-32}$ and $J$ is the spectral overlap $^{27}$. Förster and Dexter electronic energy transfer mechanisms coexist for singlet-singlet energy transfer. The Förster term has $\sim 1 / r^{3}$ distance dependence and it is long range, $r>10 \AA$, while and the Dexter term has $\sim \exp (-\beta r)$ distance dependence and it is short range, $r<10 \AA^{27}$.

Equation 2 reports the transfer rate constant for a CT process ${ }^{27}$ where $V_{C T}$ is the electronic coupling matrix element term, $\lambda$ is the total reorganization energy and $\Delta \mathrm{G}$ is the free energy difference term between reactants and products ${ }^{27,33}$. Details about the EET and CT parameters used in equations 1 and 2 are reported and discussed in the Supplementary Information (S.5).

Figure 6 reports both EET (blue line) and CT (red line) transfer rates (equations 1 and 2) for a selection of parameters (see Supplementary Information for details) in a time window from $f_{\mathrm{s}}$ to $m s$. 


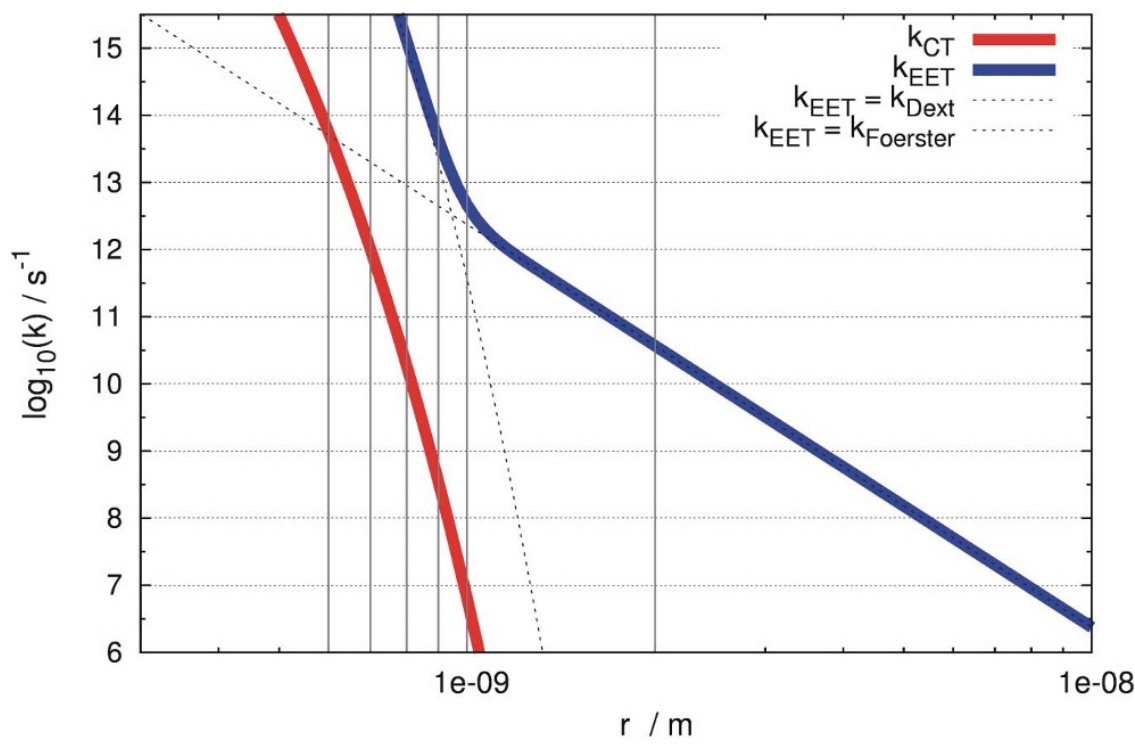

Figure 6 Charge and Energy transfer rate calculations. Electronic energy transfer (EET) transfer rate (blue line, eq. 1) and charge transfer (CT) rate (red line, eq. 2) as a function of the donor-acceptor distance $r$ (logarithmic scale). Dotted lines represent the Förster like $\left(\sim 1 / r^{6}\right)$ and the Dexter like $\left(\sim \exp (-\beta r)^{2}\right)$ contribution to the EET transfer rate. Details about EET and CT parameters present in eq. 1 and 2 are reported in Supplementary Information (Section S5).

Considering the P3HT/PCBM interface as molecular in nature and, as Density Functional Theory (DFT) calculations ${ }^{34,35}$, provide optimized intermolecular distance between the P3HT and PCBM centers around 6-9 $\AA$, we focus the transfer rate analysis on the 6 $\AA$ to $1 \mathrm{~nm}$ spatial range. As reported in Figure 6, in this spatial range EET is faster than CT mechanism and the time scales obtained are in good agreement with those observed in the experiments, with EET in the ultrafast time regime (from few fs to hundreds of fs, Figure 3 ) and the CT, or the charge separation process, in the picosecond time scales $^{8,36-39}$. The short range EET is due to Dexter coupling that out-competes both Förster and CT. According to the theory developed by Scholes and Ghiggino ${ }^{28-31}$, the Dexter exchange integral can be well represented as a through-configuration exciton resonance interaction term where the overlap integrals between neutral-excited- $\left(\Psi_{1}\left(\mathrm{D}^{*} / \mathrm{A}\right), \Psi_{4}\left(\mathrm{D} / \mathrm{A}^{*}\right)\right)$ and ionic-like $\left(\Psi_{2}\left(\mathrm{D}^{+} / \mathrm{A}^{-}\right)\right.$, $\Psi_{3}\left(\mathrm{D}^{-} / \mathrm{A}^{+}\right)$) electronic wavefunctions play a major role, giving the short range dependence contribution. Due to interface level alignment of type II, amongst ionic-like configurations, only $\Psi_{2}$ is however expected to play a major role in the overall wavefunction superposition ${ }^{30}$.

Calculations based on such equations support the experimental observation that EET is faster than CT (at the molecular level). Here the equations 1 and 2 are used just to support the experimental evidence of an ultrafast EET mechanism, without pretending to extend this physical scenario to the whole range of D/A distances and for any kind of D/A blend morphologies.

In conclusion we report here the dynamics occurring in the finely intermixed phase of P3HT and PCBM. We show that the expected charge generation process is mediated by an initial ultrafast electronic energy transfer from the polymer to the acceptor molecule. The initial energy transfer $\left(D^{*}+A \rightarrow D+A^{*}\right)$ takes place in less than $200 \mathrm{fs}$. Charge separation is a secondary process occurring between the excited acceptor and the polymer domain on the $100 \mathrm{ps}$ time scale. Such intermixed domains are the actual border regions between $\mathrm{D}$ and $\mathrm{A}$ clusters in the photovoltaic active layer based on BHJ. In this frame electronic energy transfer should be an important event in the primarily step for charge generation, usually underestimated in the description of the photovoltaic event. This, first of all, brings to some considerations on the role and actual values assigned so far to the exciton diffusion length. The critical length scale of $10 \mathrm{~nm}$ we found here is actually the convolution among the exciton diffusion, the molecular inter-diffusion and the average distance which allow for electronic energy transfer at the interface. Finally we note that Ward et al. ${ }^{15}$ have recently shown a possible energy transfer pathway from copolymers such as PCDTDBT to PCBM. Thus, the process we highlight here is not necessarily specific to P3HT/PCBM, but it may be considered as an unavoidable paradigm for the concept of organic photovoltaic.

\section{Methods}

Sample preparation. The poly(3-hexylthiophene) (P3HT) (Merk, >99\% regioregular) was diluted in a dichlorobenzene (DCB) solution at different weighting ratio $(1 \mathrm{mg} / \mathrm{mL}, 5 \mathrm{mg} / \mathrm{mL})$ and heated at $70^{\circ} \mathrm{C}$ degrees for 1 hour. The solution was spin casted on a clean $250 \mu \mathrm{m}$ thick fused silica substrate at $4000 \mathrm{rpm}$ or $1000 \mathrm{rpm}$ for 45 seconds, respectively, in order to get a film thickness of 5 or $50 \mathrm{~nm}$. Note that the freshly-spun P3HT films have been dried on a hotplate at $70^{\circ}$ for $10 \mathrm{~min}$ prior to spin-coating the top layer. For the production of the bilayer we adopt the spin-coating sequential approach using an orthogonal solvent combination (dichlorobenzene and dichloromethane) to process the [6,6]-phenyl C61-butyric acid methyl ester (PCBM) layer on top of P3HT preventing the re-dissolution of the P3HT underneath. The PCBM was dissolved in a dichloromethane solution $(20 \mathrm{mg} / \mathrm{mL})$, briefly heated at $40^{\circ} \mathrm{C}$ to ensure maximal dissolution. The PCBM solution was casted at $4000 \mathrm{rpm}$ for 45 seconds onto the $\mathrm{P} 3 \mathrm{HT}$ layer to obtain a thickness of $5 \mathrm{~nm}$. In order to increase the order of the P3HT layer before PCBM deposition we perform a thermal annealing, heating the sample to 140 degrees for 5 minutes.

TrAMP. For the ultrafast experiments, laser pulses (40 fs, $250 \mathrm{kHz}$ ) at $800 \mathrm{~nm}$ were generated using Coherent Micra Ti:Saphire oscillator in conjunction with Coherent RegA 9040 amplifier. Part of the laser output was used to pump Coherent OPA in order to generate pump pulsed at $520 \mathrm{~nm}$. The other part of the pulse was used to pump a sapphire plate $(2 \mathrm{~mm})$ to generate white light continuum as the probe pulse. The delay between the pump and probe pulses was controlled by a computer controlled translation stage. Both the pulses were made collinear using a beam splitter before launching into the TrAMP setup.

The TrAMP system can be thought to be a misaligned concentric optical cavity; as shown in figure 1 , one of the mirrors focuses the beam and the second mirror collimates the beam and sends it back. At proper incidence conditions, a multireflection can be achieved between the two mirrors with the ray tracing an ellipse on the sample (as well as on the mirrors). The number of reflections can be controlled by tuning the angle of incidence and the mirror alignment or alternatively, the beam can be extracted from the cavity using another smaller mirror.

A high pass filter was used to remove the pump pulse; the probe pulse was dispersed using a monochromator and detected using a lock-in, photo-diode system.

1. Howard, I. A., Mauer, R., Meister, M. \& Laquai, F. Effect of Morphology on Ultrafast Free Carrier Generation in Polythiophene:Fullerene Organic Solar Cells. J. Am. Chem. Soc. 132, 14866-14876 (2010). 
2. Ma, W. et al. Thermally stable efficient polymer solcar cells with nanoscale control of the interpenetrating network morphology. Adv. Funct. Mater. 15, 1617-1622 (2005).

3. Erb, T. et al. Correlation between structural and optical properties of composite polymer/fullerene films for organic solar cells. Adv. Funct. Mater. 15, 1193-1196 (2005).

4. Yang, X. et al. Nanoscale morphology of high performance polymer solar cells. Nano Lett. 5, 579-583 (2005).

5. Dennler, G., Scharber, M. C. \& Brabec, C. J. Polymer-Fullerene bulk-hetrojuction solar cells. Adv. Mater. 21, 1323-1338 (2009).

6. Savenije, T., Kroeze, J. E., Yang, X. \& Loos, J. The effect of thermal treatment on the morphology and charge carrier dynamics in a polythiophene-fullerere bulk heterojuctions. Adv, Funct. Mater. 15, 1260-1266 (2005).

7. Rochester, C. W., Mauger, S. C. \& Moulé, A. J. Investigating the morphology of polymer/fullerene layers coated using orthogonal solvents. J. Phys. Chem. C. 116, 7287-7292 (2012)

8. Ohkita, H. et al. Charge Carrier Formation in Polythiophene/Fullerene Blend Films Studied by Transient Absorption Spectroscopy. J. Am. Chem. Soc. 130(10), 3030-3042 (2008)

9. Guo, J., Ohkita, H., Benten, H. \& Ito, S. Charge Generation and Recombination Dynamics in Poly(3-hexylthiophene)/Fullerene Blend Films with Different Regioregularities and Morphologies. J. Am. Chem. Soc. 132, 6154-6164 (2010).

10. Coffey, D. C., Ferguson, A. J., Kopidakis, N. \& Rumbles, G. Photovoltaic charge generation in organic semiconductors based on long range energy transfer. ACS Nano. 4, 5437-5445 (2010)

11. Liu, Y. X., Summers, M. A., Scully, S. R. \& McGehee, M. D. Resonance energy transfer from organic chromophores to fullerene molecules. J. Appl. Phys. 99, 093521 (2006).

12. Scully, S. R., Armstrong, P. B., Edder, C., Fréchet, J. M. J. \& McGehee, M. D. Long range resonant energy transfer for enhanced exciton harvesting for organic solar cells. Adv. Mater. 19, 2961-2966 (2007).

13. van Hal, P. A. et al. Two-step mechanism for photoinduced intramolecular electron transfer in oligo(p-phenylene vinylene)-fullerene dyads. Phy. Rev. B. 64, 075206 (2001)

14. Ruseckas, A., Shaw, P. E. \& Samuel, I. D. W. Probing the nanoscale phase separation in binary photovoltaic blends of poly(3-hexulthiophene) and methanofullerene by energy transfer. Dalton Trans 45, 10040-10043 (2009).

15. Ward, A. J., Ruseckas, A. \& Samuel, I. D. W. A shift from diffusion assisted to energy transfer controlled fluorescence quenching in polymer-fullerene photovoltaic blends. J. Phys. Chem. C. 116(45), 23931-23937 (2012)

16. Herriott, D., Kogelnik, H. \& Kompfner, R. Off-Axis Paths in Spherical Mirror Interferometers. Applied Optics. 3, 523-526 (1964).

17. Chen, H., Hegde, R., Browning, J. \& Dadmun, M. D. The miscibility and depth profile of PCBM in P3HT: thermodynamic information to improve organic photovoltaics. Phys. Chem. Chem. Phys. 14, 5635-5641 (2012).

18. Korovyanko, O. J. et al. Photoexcitation dynamincs in regioregular and regiorandom polythiophene films. Phys. Rev. B. 64, 235122 (2001).

19. Cabanillas-Gonzalez, J., Grancini, G. \& Lanzani, G. Pump-probe spectroscopy in Organic semiconductors: Monitoring Fundamental Processes of Relevance in Optoelectronics. Adv Mater. 23, 5468-5485 (2011).

20. Lanzani, G. Editor. Photophysics of Molecular Materials, Wiley VCH Verlag $\mathrm{GmbH}(2006)$

21. Bakulin, A. A., Hummelen, J. C., Pshenichnikov, M. S. \& van Loosdrecht, H. M. Ultrafast Hole-transfer dynamics in Polymer/PCBM bulk heterojunctions. Adv. Funct. Mater. 20, 1653-1660 (2010).

22. Pillai, S. et al. Carotenoids as electron or excited state enegy donors in artificial photosynthesis: an ultrafast investigation of a carotenoporphyrin and carotefullerene dyad. Phys. Chem. Chem. Phys. 15, 4775-4784 (2013).

23. Grancini, G. et al. Transient Absorption Imaging of P3HT:PCBM Photovoltaic Blend: Evidence for Interfacial Charge Transfer State. J Phys. Chem. Lett. 2, 1099-1105 (2011).

24. Scharber, M. C. et al. Design rules in bulk-heterojuction solar cells - towards $10 \%$ energy-conversion efficiency. Adv. Mater. 18, 789-794 (2006).

25 . Singh, S. et al. Two-step charge photogeneration dynamics in polymer/fullerene blends for photovoltaic applications. Phys. Rev. B. 83, 155124 (2012).
26. Olaya-Castro, A. \& Scholes, G. D. Energy transfer from Forster-Dexter theory to quantum coherent light harvesting. Int. Rev. Phys. Chem. 30, 49-77 (2011)

27. Ito, A. \& Meyer, T. J. The Golden rule. Application for fun and profit in electron transfer, energy transfer and excited state decay. Phys. Chem. Chem. Phys. 14, 13731-13745 (2012).

28. Scholes, G. D. Long range resonance energy transfer in molecular systems. Annu. Rev. Phys. Chem. 54, 57 (2003).

29. Scholes, G. D. \& Ghiggino, K. P. Rate expressions for excitation transfer I. Radiationless transition theory perspective. J. Chem. Phys. 101, 1251 (1994).

30. Harcourt, R. D., Scholes, G. D. \& Ghiggino, K. P. Rate expressions for excitation transfer II. Electronic considerations of direct and through-configuration exciton resonance interactions. J. Chem. Phys. 101, 10521 (1994)

31. Scholes, G. D., Harcourt, R. D. \& Ghiggino, K. P. Rate expressions for excitation transfer III. An ab-initio study of electronic factors in excitation transfer and exciton resonance interactions. J. Chem. Phys. 102, 9574 (1995).

32. Scholes, G. D. \& Ghiggino, K. P. Rate expressions for excitation transfer. IV. Energy migration and superexchange phenomena. J. Chem. Phys. 103, 8873 (1995).

33. Nitzan, A. Chemical Dynamics in Condesed Phases, Oxford University Press (2011).

34. Grancini, G. et al. Hot exciton dissociation in polymer solar cells. Nat. Mater. 12, 29-33 (2013).

35. Sen, K., Crespo-Otero, R., Weingart, O., Thiel, W. \& Barbatti, M. Interfacial states in donor-acceptor organic heterojuctions: computational insights into thiopheneoligomer/fullerene junctions. J. Chem. Theory. Comput. 9, 533-543 (2013).

36. Liu, T. \& Troisi, A. Absolute rate of charge separation and recombination in a molecular model of the P3HT/PCBM interface. J. Phys. Chem. C. 115, 2406-2415 (2011).

37. Ai, X. et al. Photoinduced charge carrier generation in a poly (3-hexylthiophene) and methanofullerene bulk heterojunction investigated by time-resolved terahertz spectroscopy. J. Phys. Chem. B. 110, 25462-25471 (2006).

38. Choi, J. H. et al. Thienyl-substituted methanofullerene derivatives for organic photovoltaic cells. J. Mater. Chem. 20, 475-482 (2010).

39. Piris, J. et al. Photogeneration and ultrafast dynamics of excitons and charges in P3HT/PCBM blends. J. Phys. Chem. C. 113, 14500-14506 (2009).

\section{Acknowledgements}

ARSK and GL acknowledge the financial support of the Marie-Curie grant under the FP7 project PITNGA-2009-237900(ICARUS). The authors also thank Simone Guarnera, Dario Polli and Luca Frezza for help and discussions. The authors also acknowledge the financial support from Italian Ministry of University Research (project PRIN 2010-2011 "DSSCX", Contract 20104XET32) and POCAONTAS (Grant agreement No: 316633) is also acknowledged.

\section{Author contributions}

The experimental set-up was developed by A.R.S.K.; the sample was prepared by G.G., A.R.S.K. and S.P. The measurements were done by A.R.S.K. and S.S.K.R. The data have been analyzed by A.R.S.K., G.G., A.P. and G.L. The experiment was conceived by G.L., A.P. and A.R.S.K. Theoretical calculations have been carried out by D.F. All the authors contributed to writing of the paper.

\section{Additional information}

Supplementary information accompanies this paper at http://www.nature.com/ scientificreports

Competing financial interests: The authors declare no competing financial interests How to cite this article: Kandada, A.R.S. et al. Ultrafast Energy Transfer in Ultrathin Organic Donor/Acceptor Blend. Sci. Rep. 3, 2073; DOI:10.1038/srep02073 (2013).

This work is licensed under a Creative Commons AttributionNonCommercial-NoDerivs 3.0 Unported license. To view a copy of this license, visit http://creativecommons.org/licenses/by-nc-nd/3.0 